\title{
Ultra-processed food intake and mortality in the USA: results from the Third National Health and Nutrition Examination Survey (NHANES III, 1988-1994)
}

\author{
Hyunju Kim ${ }^{1,2}$, Emily A Hu ${ }^{2,3}$ and Casey M Rebholz ${ }^{2,3, *}$ \\ ${ }^{1}$ Center for Human Nutrition, Johns Hopkins Bloomberg School of Public Health, Baltimore, MD, USA: ${ }^{2}$ Welch Center \\ for Prevention, Epidemiology and Clinical Research, Department of Epidemiology, Johns Hopkins Bloomberg School \\ of Public Health, 2024 East Monument Street, Suite 2-500, Baltimore, MD 21287 , USA: ${ }^{3}$ Department of \\ Epidemiology, Johns Hopkins Bloomberg School of Public Health, Baltimore, MD, USA
}

Submitted 13 August 2018: Final revision received 22 November 2018: Accepted 5 December 2018: First published online 21 February 2019

\begin{abstract}
Objective: To evaluate the association between ultra-processed food intake and all-cause mortality and CVD mortality in a nationally representative sample of US adults.

Design: Prospective analyses of reported frequency of ultra-processed food intake in 1988-1994 and all-cause mortality and CVD mortality through 2011.

Setting: The Third National Health and Nutrition Examination Survey (NHANES III, 1988-1994).

Participants: Adults aged $\geq 20$ years ( $n$ 11898).

Results: Over a median follow-up of 19 years, individuals in the highest quartile of frequency of ultra-processed food intake (e.g. sugar-sweetened or artificially sweetened beverages, sweetened milk, sausage or other reconstructed meats, sweetened cereals, confectionery, desserts) had a $31 \%$ higher risk of all-cause mortality, after adjusting for demographic and socio-economic confounders and health behaviours (adjusted hazard ratio $=1.31 ; 95 \% \mathrm{CI} 1.09,1.58 ; P$-trend $=$ 0.001). No association with CVD mortality was observed $(P$-trend $=0 \cdot 86$ ).

Conclusions: Higher frequency of ultra-processed food intake was associated with higher risk of all-cause mortality in a representative sample of US adults. More longitudinal studies with dietary data reflecting the modern food supply are needed to confirm our results.
\end{abstract}

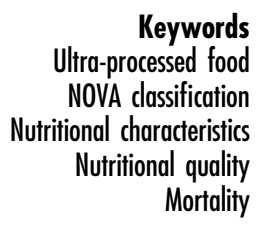

In the last few decades, the global food system has changed significantly. Ultra-processed foods, characterized as ready-to-eat foods that are manufactured with substances from industrial processing (chemical additives, colorants, flavourings), are more readily available in the food supplies of high- and middle-income countries ${ }^{(1)}$. Recent cross-sectional studies using individual-level consumption data have reported that ultra-processed foods contribute $25-60 \%$ of total energy intake in the USA ${ }^{(2)}$, Canada $^{(3)}$, Brazil $^{(4)}$, France ${ }^{(5)}$, Mexico $^{(6)}$ and Chile ${ }^{(7)}$. In the USA, these foods account for a strikingly high percentage (90\%) of the energy from added sugar ${ }^{(2)}$.

High consumption of ultra-processed foods raises health concerns. As ultra-processed foods contain little or no whole foods, these foods are nutritionally poor, with high amounts of fat, added sugar and energy, and low amounts of micronutrients and fibre ${ }^{(2,3,5,8)}$. A systematic review reported a direct association between these types of foods, such as sugar-sweetened beverages or sweetened/salted snacks, and body fat in children and adolescents ${ }^{(9)}$. In addition to low nutritional quality, ultraprocessed foods and their packaging contain compounds that can pose health risks ${ }^{(10-12)}$. Compounds such as acrylamide, polycyclic aromatic hydrocarbons, heterocyclic amines and furans may be present in ultraprocessed foods as they can be generated during heating, a commonly used food processing technique ${ }^{(13,14)}$. Exposure to chemicals such as phthalates and bisphenol A, which are used in food packaging as plasticizers, is also possible ${ }^{(15,16)}$.

Furthermore, previous studies which classified foods using predefined criteria on degree of processing have shown elevated risk of chronic diseases with high consumption of ultra-processed foods. A longitudinal study 
of Brazilian children showed that ultra-processed food intake was associated with dyslipidaemia ${ }^{(17)}$. In adults, cross-sectional studies have shown an increased odds of obesity with higher consumption of ultra-processed foods ${ }^{(18-20)}$. A prospective cohort of university students in Spain reported a dose-response relationship between ultra-processed food consumption and a higher risk of incident obesity and hypertension ${ }^{(21,22)}$. In addition, a recent population-based study from France found that a $10 \%$ increase in ultra-processed foods in the diet was associated with a higher risk of overall cancer and breast cancer $^{(23)}$. These studies suggest that there are adverse health outcomes associated with ultra-processed food intake, but all of these studies are limited in that they are either cross-sectional or had a relatively short follow-up period (5-9 years).

To address these gaps, we investigated the association between ultra-processed food intake and all-cause mortality and CVD mortality using data from a nationally representative sample of US adults with over 20 years of follow-up. We focused on CVD mortality given the previously reported associations between ultra-processed foods and CVD risk factors ${ }^{(21,22)}$. We also aimed to examine sociodemographic and nutritional characteristics according to different levels of ultra-processed food consumption.

\section{Methods}

\section{Study population}

We conducted prospective analyses on adult participants (aged $\geq 20$ years) from the Third National Health and Nutrition Examination Survey (NHANES III, 1988-1994). NHANES III used a multistage, stratified, clustered, probability sampling design to identify a nationally representative sample of non-institutionalized civilians in the USA ${ }^{(24)}$. Participants completed a household interview, laboratory measurements and physical examinations ${ }^{(24)}$. Details of the survey design have been published previously ${ }^{(24)}$.

In the present study, we included adults with no missing information on ultra-processed food intake and mortality ( $n$ 18779). Since dietary intake often changes after diagnosis of a clinical condition, we excluded adults with a chronic disease, such as heart disease (self-reported diagnosis of heart attack, stroke or IHD; $n$ 1728), cancer (self-reported diagnosis of cancer; $n$ 609), diabetes (self-reported diagnosis of diabetes, diabetes medication use or fasting glucose $\geq 126 \mathrm{mg} / \mathrm{dl} ; n$ 3701) or chronic kidney disease (estimated glomerular filtration rate (eGFR) $<60 \mathrm{ml} / \mathrm{min}$ per $1.73 \mathrm{~m}^{2} ; n$ 843), at baseline. The final sample size was 11898 (see online supplementary material, Supplemental Fig. 1).

\section{Dietary assessment and classification of ultra-processed foods}

At baseline, trained interviewers administered an eightyone-item FFQ, which assessed participants' usual intakes of foods and beverages consumed in the past month, and a $24 \mathrm{~h}$ dietary recall ${ }^{(24)}$. Since portion size was not assessed on the FFQ, we used data from participants' $24 \mathrm{~h}$ dietary recalls to examine intakes of total energy, macronutrients and micronutrients. The NHANES III Nutrition Methodology Working group, which is comprised of a group of experts from academia, government agencies and industry, recommended the use of an FFQ to rank participants by their food intake ${ }^{(24)}$.

We used the NOVA classification to categorize each reported food item on the FFQ into one of the following categories representing levels of processing: (i) fresh or minimally processed foods; (ii) processed culinary ingredients; (iii) processed foods with salt, sugar or oil; or (iv) ultra-processed foods containing predominantly industrial substances and few whole foods (see online supplementary material, Supplemental Table 1$)^{(3,25,26)}$. The first category represents foods that are obtained directly from plants or animals that have undergone alterations to increase shelf life or storage. These types of alterations can range from removing inedible parts, refrigerating, freezing, vacuum packaging, drying, crushing, grinding, filtering to pasteurizing. The second category represents substances that are extracted from nature or foods from the first category, which may be used in the kitchen as culinary ingredients. The third category represents foods that have undergone simple alterations such as adding oil, sugar, salt or other items from the second category to foods in the first category. These alterations are usually made to improve sensorial qualities or extend the durability of foods in the first category. The fourth category represents foods containing substances that are not typically used in culinary preparation such as hydrogenated oils, hydrolysed protein or emulsifiers, and have few whole foods. Ultra-processed foods contain industrial substances to mimic sensorial qualities of whole foods and create foods that are highly palatable.

The focus of our study was on the fourth level of processing. After food items were classified by NOVA category, we summed the frequency of ultra-processed food intake per day for each participant and then divided the participants into quartiles based on their frequency of consumption per day.

\section{Nutrient analyses}

We used three methods to examine the nutritional characteristics of individuals reporting different frequencies of ultra-processed food consumption. For all methods, we used participants' $24 \mathrm{~h}$ dietary recall data. First, we examined nutrient density across quartiles of frequency of 
ultra-processed food intake. We expressed macronutrients (protein, carbohydrates, total fat, saturated fat, monounsaturated fat, polyunsaturated fat) and added sugars as percentage of energy, and fibre, cholesterol and micronutrients as grams, milligrams or micrograms per $4184 \mathrm{~kJ}$ (1000 kcal). All nutrient estimates except for added sugars were assigned by the National Center for Health Statistics using the US Department of Agriculture food composition database $^{(24)}$. To derive estimates on added sugar intake, we used the Pyramid Servings Database developed by the National Cancer Institute. This database provides estimates of added sugars in all food items reported in NHANES III $24 \mathrm{~h}$ dietary recalls ${ }^{(27)}$.

Second, we used the Nutrient-Rich Foods (NRF) index, a score which ranks the nutritional quality of a food. NRF score has been associated with overall diet quality ${ }^{(28)}$ and has been inversely associated with all-cause mortality in a European cohort $^{(29)}$. Details on development, validation and calculation of the score have been published previously $^{(28,30)}$. Briefly, NRF9.3 is calculated based on nine nutrients to encourage (protein, fibre, vitamins A, C and E, $\mathrm{Ca}, \mathrm{Fe}, \mathrm{Mg}$ and $\mathrm{K}$ ) and three nutrients to limit (saturated fat, added sugar and Na). A higher NRF9.3 food score indicates higher nutritional quality of a food. We additionally excluded pregnant and lactating women for this analysis ( $n$ 267), as micronutrient intakes differ during this period. For each participant, we summed the NRF9.3 food scores, divided by total energy intake, and expressed the NRF9. 3 index score per $418 \mathrm{~kJ}$ ( $100 \mathrm{kcal})$.

Lastly, we used the Healthy Eating Index (HEI)-2000, a 100-point score based on the US Dietary Guidelines for Americans ${ }^{(31)}$, to assess diet quality across quartiles of ultra-processed food intake.

\section{Outcome ascertainment}

From baseline through 31 December 2011, participants' vital status and cause-of-death information were followed by the National Center for Health Statistics. Vital status was determined by probabilistic matching of participants to the National Death Index based on identifying information, including social security number, name, sex and date of birth $^{(32)}$. Thus, only participants with insufficient information on these matching criteria were lost to follow-up. Details of the linkage methods have been reported previously ${ }^{(32)}$. We calculated follow-up time (number of days) as the time from NHANES examination until the date of death or the end of follow-up on 31 December 2011. We defined death due to CVD as those with a primary cause of death listed as I00-I69 in the International Classification of Diseases and Related Health Problems, 10th edition ${ }^{(33)}$.

\section{Covariates}

Participants self-reported the following covariates: age (continuous), sex (male or female), race/ethnicity (nonHispanic White; non-Hispanic Black; Mexican American; other), total energy intake (continuous), education level (less than high school; high school; more than high school), income (poverty-income ratio: $<130 \%$; $130-<350 \%$; $\geq 350 \%$ ), health behaviours including smoking (current smoker; former smoker; never smoker), alcohol consumption (quartiles) and physical activity (continuous).

Participants reported frequency of engaging in any moderate or vigorous physical activity such as walking, jogging, running, bicycling, swimming, aerobics, lifting weights, dancing or gardening in the past week. For each activity, an intensity rating (a metabolic equivalent of task, MET) was assigned ${ }^{(34)}$. We multiplied the frequency and intensity of any physical activity performed in the past week.

Participants' height (centimetres) and weight (kilograms) were measured using standardized methods ${ }^{(24)}$. We calculated BMI from this information and categorized it as underweight $\left(<18.5 \mathrm{~kg} / \mathrm{m}^{2}\right)$, normal weight $\left(18.5-<25.0 \mathrm{~kg} / \mathrm{m}^{2}\right)$, overweight $\left(25.0-<30.0 \mathrm{~kg} / \mathrm{m}^{2}\right)$ or obese $\left(\geq 30 \cdot 0 \mathrm{~kg} / \mathrm{m}^{2}\right)$. Baseline hypertension was defined as systolic blood pressure $\geq 140 \mathrm{mmHg}$, diastolic blood pressure $\geq 90 \mathrm{mmHg}$ or self-reported use of antihypertensive medication. Serum total cholesterol was assessed using enzymatic methods that were previously described $^{(35)}$. We calculated eGFR using the 2009 Chronic Kidney Disease Epidemiology Collaboration equation after calibrating serum creatinine measurements to reference values at the Cleveland Clinic Research Laboratory ${ }^{(36,37)}$. We used serum total cholesterol and eGFR as continuous variables. The percentages of participants with missing covariates were low (range: $0-8 \%$ ). We conducted the analyses among participants with no missing information.

\section{Statistical analyses}

We examined participants' baseline characteristics and nutritional characteristics according to quartiles of frequency of ultra-processed food intake. We used weighted $\chi^{2}$ tests for categorical variables and weighted ANOVA for continuous variables ${ }^{(38)}$.

We performed three Cox proportional hazards models, with length of follow-up time as the time metric, to calculate hazard ratios (HR) and $95 \%$ CI for the association between frequency of ultra-processed food intake and allcause mortality and CVD mortality. Model 1 adjusted for demographic characteristics (age, sex, race/ethnicity) and total energy intake. Model 2 adjusted for covariates in Model 1 as well as socio-economic factors (poverty level, education level) and health behaviours (smoking status, physical activity, alcohol intake). In order to test the mediating effect of BMI, hypertension status, total cholesterol and eGFR, secondary analysis was carried out adjusting for these variables in addition to covariates in Model 2 (Model 3). We considered Model 2 as our main result because Model 3 includes factors that may be along 
Table 1 Baseline participant characteristics, according to quartile of frequency of ultra-processed food intake, of adults aged $\geq 20$ years ( $n$ 11 898), Third National Health and Nutrition Examination Survey (NHANES III, 1988-1994)*

\begin{tabular}{|c|c|c|c|c|c|c|c|c|c|}
\hline \multirow[b]{3}{*}{ Characteristic } & \multicolumn{8}{|c|}{ Quartile of frequency of ultra-processed food intake } & \multirow[b]{3}{*}{$P$ value } \\
\hline & \multicolumn{2}{|c|}{ Quartile 1 ( $n$ 2982) } & \multicolumn{2}{|c|}{ Quartile 2 (n 2989) } & \multicolumn{2}{|c|}{ Quartile 3 ( $n$ 2985) } & \multicolumn{2}{|c|}{ Quartile 4 (n 2942) } & \\
\hline & Mean & SE & Mean & $\mathrm{SE}$ & Mean & SE & Mean & $\mathrm{SE}$ & \\
\hline Ultra-processed food intake (times/d), range & \multicolumn{2}{|c|}{$0-<2.6$} & \multicolumn{2}{|c|}{$2.6-<3.8$} & \multicolumn{2}{|c|}{$3 \cdot 8-<5 \cdot 2$} & \multicolumn{2}{|c|}{$5 \cdot 2-<29 \cdot 8$} & $<0.001$ \\
\hline Age (years) & 43 & 0.5 & 42 & 0.5 & 41 & 0.5 & 38 & 0.5 & $<0.001$ \\
\hline Female sex $(\%)$ & \multicolumn{2}{|c|}{$\begin{array}{l}43 \\
59\end{array}$} & \multirow{2}{*}{\multicolumn{2}{|c|}{52}} & \multicolumn{2}{|c|}{51} & \multirow{2}{*}{\multicolumn{2}{|c|}{45}} & $<0.001$ \\
\hline \multicolumn{8}{|l|}{ Race/ethnicity (\%) } & & \\
\hline Non-Hispanic White & \multicolumn{2}{|c|}{65} & \multicolumn{2}{|c|}{77} & & & \multicolumn{2}{|c|}{80} & $<0.001$ \\
\hline Non-Hispanic Black & \multicolumn{2}{|c|}{11} & \multicolumn{2}{|c|}{9} & & & \multicolumn{2}{|c|}{12} & \\
\hline Mexican American & \multicolumn{2}{|c|}{8} & \multicolumn{2}{|c|}{6} & & & \multicolumn{2}{|c|}{4} & \\
\hline Other & \multirow{2}{*}{\multicolumn{2}{|c|}{16}} & \multicolumn{2}{|c|}{8} & & & \multicolumn{2}{|c|}{4} & \\
\hline \multicolumn{8}{|l|}{ Poverty level (\%) } & & \\
\hline$<130 \%$ & \multicolumn{2}{|c|}{20} & & & & & & & $<0.001$ \\
\hline $130-<350 \%$ & & & & & & & & & \\
\hline$\geq 350 \%$ & & & & & & & & & \\
\hline Education level (\%) & & & & & & & & & \\
\hline Less than high school & & & & & & & & & $<0.001$ \\
\hline High school & & & & & & & & & \\
\hline More than high school & & & & & & & & & \\
\hline Smoking status (\%) & & & & & & & & & \\
\hline Current & & & & & & & & & 0.01 \\
\hline Former & & & & & & & & & \\
\hline Never & & & & & & & & & \\
\hline Physical activity (MET/week) & 29 & 5.5 & 24 & $1 \cdot 0$ & 25 & 0.9 & 27 & 0.9 & 0.08 \\
\hline Alcohol intake (drinks/month) & 8 & 0.6 & 9 & 0.6 & 9 & 0.4 & 10 & 0.7 & 0.09 \\
\hline BMI $\left(\mathrm{kg} / \mathrm{m}^{2}\right)$ & $26 \cdot 2$ & $0 \cdot 1$ & $26 \cdot 3$ & 0.1 & $26 \cdot 1$ & 0.2 & $26 \cdot 2$ & 0.2 & 0.95 \\
\hline Hypertension status (\%) & & & & & & & & & 0.71 \\
\hline Total cholesterol (mg/dl) & 203 & 1.5 & 202 & 1.2 & 201 & 1.3 & 197 & 1.4 & 0.02 \\
\hline eGFR $\left(\mathrm{ml} / \mathrm{min}\right.$ per $\left.1.73 \mathrm{~m}^{2}\right)$ & 101 & 0.5 & 102 & 0.6 & 102 & 0.6 & 105 & 0.5 & $<0.001$ \\
\hline
\end{tabular}

MET, metabolic equivalent of task; eGFR, estimated glomerular filtration rate.

*Values presented are means and their SE for continuous variables, and as percentages for categorical variables.

†We tested for differences in baseline characteristics using weighted $x^{2}$ tests for categorical variables and weighted ANOVA for continuous variables.

the causal pathway. We tested for a linear trend across quartiles using the median value within each quartile.

As sensitivity analyses, we additionally controlled for diet quality scores, as ultra-processed food consumption can be a marker of an unhealthy dietary pattern and to examine the mediating role of diet quality on the association between ultra-processed foods and mortality. In addition, we excluded the first two years of follow-up for all participants to minimize the possibility that deaths were due to an underlying health condition at baseline. We repeated the analyses excluding processed meats (bacon, sausage and processed meats) from ultra-processed food intake to confirm that the observed associations were not due to consumption of processed meat, which has a strong and direct association with mortality ${ }^{(39)}$. Analyses were conducted using the statistical software package Stata version 13.0.

\section{Results}

In the overall sample, participants consumed ultraprocessed foods a mean of 4 times/d (range: 0-29.8 times/d). A total of $0.06 \%$ of the analytic sample consumed no ultra-processed food on a daily basis. Those in the highest quartile consumed ultra-processed foods more than 5 times/d.
Those in the highest quartile of frequency of ultraprocessed food intake were more likely to be younger, male, non-Hispanic White and current smokers, and were less likely to have less than a high school education or to have a household income more than $350 \%$ of poverty level ( $P \leq 0.01$ for all comparisons; Table 1$)$. Those in the highest quartile were more likely to have lower serum total cholesterol $(P=0 \cdot 02)$ and higher eGFR $(P<0 \cdot 001)$.

Participants in the highest quartile of frequency of ultraprocessed food intake had significantly higher intakes of total energy, total fat, saturated fat, monounsaturated fat and added sugar, and lower intake of protein $(P<0.001$ for all comparisons; Table 2). Intakes of fibre, cholesterol and almost all micronutrients were significantly lower among participants in the highest quartile. Those in the highest quartile had lower scores for nutritional quality using NRF9.3 $(P<0 \cdot 001)$ and diet quality using HEI-2000 $(P=0 \cdot 001)$.

Over a median follow-up of 19 years, 2451 deaths due to any cause occurred and 648 deaths were due to CVD. In Model 2, those in the highest quartile of frequency of ultraprocessed food had a $31 \%$ higher risk of death compared with those in the lowest quartile (HR $=1.31 ; 95 \%$ CI 1.09, 1.58; $P$-trend across quartiles $=0.001$; Table 3). When potential mediating variables were included in the model (Model 3), the association did not change substantially $(\mathrm{HR}=1 \cdot 30 ; 95 \%$ CI 1.08, 1.57; $P$-trend $=0 \cdot 001)$. 
Table 2 Nutritional characteristics, according to quartile of frequency of ultra-processed food intake (times/d) from participants' $24 \mathrm{~h}$ dietary recalls, of adults aged $\geq 20$ years $(n 11$ 898), Third National Health and Nutrition Examination Survey (NHANES III, 1988-1994)

\begin{tabular}{|c|c|c|c|c|c|c|c|c|c|}
\hline \multirow[b]{3}{*}{ Nutrient } & \multicolumn{8}{|c|}{ Quartile of frequency of ultra-processed food intake } & \multirow[b]{3}{*}{$P$ value* } \\
\hline & \multicolumn{2}{|c|}{ Quartile 1 (n 2982) } & \multicolumn{2}{|c|}{ Quartile 2 (n 2989) } & \multicolumn{2}{|c|}{ Quartile 3 (n 2985) } & \multicolumn{2}{|c|}{ Quartile 4 (n 2942) } & \\
\hline & Mean & SE & Mean & SE & Mean & SE & Mean & SE & \\
\hline Total energy intake $(\mathrm{kJ} / \mathrm{d})$ & 8242 & $139 \cdot 3$ & 9096 & $146 \cdot 4$ & 9673 & $141 \cdot 8$ & 10765 & $142 \cdot 3$ & $<0.001$ \\
\hline Total energy intake $(\mathrm{kcal} / \mathrm{d})$ & 1970 & $33 \cdot 3$ & 2174 & $35 \cdot 0$ & 2312 & 33.9 & 2573 & $34 \cdot 0$ & $<0.001$ \\
\hline Protein (\% of energy) & $16 \cdot 2$ & $0 \cdot 2$ & $15 \cdot 2$ & 0.1 & $15 \cdot 0$ & 0.1 & $14 \cdot 7$ & $0 \cdot 1$ & $<0.001$ \\
\hline Carbohydrates (\% of energy) & $49 \cdot 3$ & 0.5 & $49 \cdot 8$ & 0.4 & 49.5 & 0.4 & 49.9 & 0.4 & 0.31 \\
\hline Fat (\% of energy) & $25 \cdot 1$ & 0.3 & $25 \cdot 9$ & 0.3 & $26 \cdot 5$ & 0.2 & $26 \cdot 7$ & 0.2 & $<0.001$ \\
\hline SFA (\% of energy) & $8 \cdot 4$ & 0.1 & $8 \cdot 7$ & $0 \cdot 1$ & 8.9 & 0.1 & $9 \cdot 1$ & 0.1 & $<0.001$ \\
\hline MUFA (\% of energy) & $9 \cdot 3$ & 0.1 & $9 \cdot 7$ & $0 \cdot 1$ & $9 \cdot 9$ & $0 \cdot 1$ & $10 \cdot 1$ & $0 \cdot 1$ & $<0.001$ \\
\hline PUFA (\% of energy) & $5 \cdot 4$ & 0.1 & $5 \cdot 5$ & $0 \cdot 1$ & $5 \cdot 7$ & $0 \cdot 1$ & 5.5 & $0 \cdot 1$ & 0.15 \\
\hline Added sugars (\% of energy) & 11.3 & 0.4 & $13 \cdot 1$ & 0.4 & 14.0 & 0.3 & $16 \cdot 4$ & 0.3 & $<0.001$ \\
\hline Fibre (g/4184 kJ (1000 kcal)) & 8.6 & 0.2 & $8 \cdot 2$ & $0 \cdot 1$ & $7 \cdot 9$ & 0.1 & $7 \cdot 1$ & $0 \cdot 1$ & $<0.001$ \\
\hline Cholesterol (mg/4184 kJ (1000 kcal)) & 137 & $3 \cdot 2$ & 124 & $2 \cdot 2$ & 126 & $2 \cdot 7$ & 124 & $2 \cdot 4$ & $<0.001$ \\
\hline $\mathrm{Na}(\mathrm{mg} / 4184 \mathrm{~kJ}(1000 \mathrm{kcal}))$ & 1702 & $23 \cdot 2$ & 1637 & $17 \cdot 8$ & 1607 & $15 \cdot 4$ & 1620 & $18 \cdot 8$ & 0.02 \\
\hline $\mathrm{P}(\mathrm{mg} / 4184 \mathrm{~kJ}(1000 \mathrm{kcal}))$ & 620 & $5 \cdot 5$ & 606 & $6 \cdot 7$ & 604 & 3.9 & 593 & $5 \cdot 0$ & 0.01 \\
\hline $\mathrm{K}(\mathrm{mg} / 4184 \mathrm{~kJ}(1000 \mathrm{kcal}))$ & 1465 & 24.7 & 1395 & 17 & 1367 & $11 \cdot 7$ & 1249 & $12 \cdot 6$ & $<0.001$ \\
\hline $\operatorname{Mg}(\mathrm{mg} / 4184 \mathrm{~kJ}(1000 \mathrm{kcal}))$ & 153 & $2 \cdot 4$ & 145 & 1.9 & 143 & $1 \cdot 1$ & 133 & 1.5 & $<0.001$ \\
\hline $\mathrm{Ca}(\mathrm{mg} / 4184$ kJ (1000 kcal)) & 382 & $7 \cdot 0$ & 390 & $7 \cdot 6$ & 395 & $5 \cdot 2$ & 383 & 6.5 & 0.10 \\
\hline $\mathrm{Fe}(\mathrm{mg} / 4184 \mathrm{~kJ}(1000 \mathrm{kcal}))$ & $7 \cdot 3$ & $0 \cdot 1$ & $7 \cdot 6$ & 0.2 & $7 \cdot 4$ & $0 \cdot 1$ & $6 \cdot 9$ & $0 \cdot 1$ & $<0.001$ \\
\hline Zn (mg/4184 kJ (1000 kcal)) & 5.5 & 0.1 & $5 \cdot 5$ & 0.1 & 5.5 & 0.1 & $5 \cdot 3$ & $0 \cdot 1$ & 0.15 \\
\hline Vitamin A ( $\mu \mathrm{g}$ RAE/4184 kJ (1000 kcal)) & 561 & $26 \cdot 1$ & 510 & $26 \cdot 6$ & 501 & 13.9 & 429 & $16 \cdot 2$ & 0.001 \\
\hline Thiamin (mg/4184 kJ (1000 kcal)) & 0.8 & 0.01 & 0.8 & 0.01 & 0.8 & 0.01 & 0.8 & 0.01 & 0.04 \\
\hline Riboflavin (mg/4184 kJ (1000 kcal)) & 0.9 & 0.01 & 0.9 & 0.01 & 0.9 & 0.01 & 0.9 & 0.01 & 0.004 \\
\hline Niacin (mg/4184 kJ (1000 kcal)) & $11 \cdot 7$ & 0.2 & 11.5 & 0.2 & 11.4 & $0 \cdot 1$ & $10 \cdot 8$ & $0 \cdot 1$ & $<0.001$ \\
\hline Vitamin $B_{6}(\mathrm{mg} / 4184 \mathrm{~kJ}(1000 \mathrm{kcal}))$ & 0.9 & 0.01 & 0.9 & 0.01 & 0.9 & 0.01 & $0 \cdot 8$ & 0.01 & $<0.001$ \\
\hline Vitamin $B_{12}(\mu \mathrm{g} / 4184 \mathrm{~kJ}(1000 \mathrm{kcal}))$ & 2.5 & $0 \cdot 1$ & 2.5 & 0.2 & $2 \cdot 3$ & $0 \cdot 1$ & $2 \cdot 2$ & $0 \cdot 1$ & 0.22 \\
\hline Vitamin C (mg/4184 kJ (1000 kcal)) & $51 \cdot 2$ & 1.2 & 51.4 & $1 . \overline{6}$ & 53.3 & 1.5 & $47 \cdot 7$ & 1.4 & 0.04 \\
\hline Vitamin E (mg ATE/4184 kJ (1000 kcal)) & 4.4 & 0.1 & $4 \cdot 3$ & $0 \cdot 1$ & 4.5 & 0.1 & $4 \cdot 2$ & $0 \cdot 1$ & 0.01 \\
\hline Folate $(\mu \mathrm{g} / 4184 \mathrm{~kJ}(1000 \mathrm{kcal}))$ & $136 \cdot 7$ & $2 \cdot 5$ & $138 \cdot 3$ & $4 \cdot 1$ & 141.4 & $2 \cdot 8$ & 123.9 & $2 \cdot 3$ & $<0.001$ \\
\hline Nutrient-Rich Foods Index† & 23.2 & 0.6 & $20 \cdot 9$ & 0.6 & $20 \cdot 2$ & 0.4 & $16 \cdot 0$ & 0.4 & $<0.001$ \\
\hline Healthy Eating Index-2000 $\ddagger$ & $63 \cdot 0$ & 0.4 & 63.9 & 0.4 & $63 \cdot 8$ & 0.4 & $62 \cdot 6$ & 0.4 & 0.001 \\
\hline
\end{tabular}

RAE, retinal activity equivalent; ATE, a-tocopherol equivalent.

*We tested for differences in nutritional characteristics using weighted ANOVA.

†After excluding pregnant and lactating women, the Nutrient-Rich Foods Index was calculated based on nine nutrients to encourage (protein, fibre, vitamin $\mathrm{A}$, vitamin $\mathrm{C}$, vitamin $\mathrm{E}, \mathrm{Ca}, \mathrm{Fe}, \mathrm{K}, \mathrm{Mg}$ ) and three nutrients to discourage (saturated fat, added sugar, $\mathrm{Na}$ ):

$\sum_{i=1}^{i=9}$ (nutrient $i /$ recommended daily value for nutrient $\left.i\right) \times 100-\sum_{i=1}^{i=3}$ (nutrient $i /$ maximum daily value for nutrient $\left.i\right) \times 100$.
$\ddagger$ Healthy Eating Index-2000 can range from 0 to 100 .

No significant associations were observed for frequency of ultra-processed food intake and CVD mortality in Model 2 or Model 3 (Table 4).

Results were similar in sensitivity analyses after additionally adjusting for diet quality scores (all-cause mortality: $P$-trend $=0.001 ;$ CVD: $P$-trend $=0.54)$, after excluding the first two years of follow-up (all-cause mortality: $P$-trend $=0 \cdot 007$; CVD: $P$-trend $=0 \cdot 88)$ and after excluding bacon, sausage and processed meats from ultra-processed food intake (all-cause mortality: $P$-trend $=0.02 ; \quad$ CVD mortality: $P$-trend $=0 \cdot 45)$ in Model 3.

\section{Discussion}

Among US adults without chronic disease at baseline, we observed a significant association between higher frequency of ultra-processed food intake and higher risk of all-cause mortality over a median follow-up of 19 years. This association remained significant after adjusting for demographic and socio-economic confounders, health behaviours and clinical factors. We found that a diet that is high in ultra-processed foods consists of a high amount of fat, added sugar and total energy and is low in overall diet quality, protein, fibre and micronutrients.

Our study builds on previous longitudinal studies which used the NOVA framework to study the association between ultra-processed food intake and chronic conditions. These studies reported adverse health outcomes in association with ultra-processed food consumption including obesity, hypertension and cancer at mid-life (40 years of age or older at follow-up) ${ }^{(21-23)}$. To the best of our knowledge, the present study is the first which examined the association between ultra-processed food intake and mortality. Our findings suggest that the adverse health risks associated with ultra-processed food consumption, which were observed in previous studies in mid-life, may lead to a shorter lifespan.

There are several pathways through which ultraprocessed foods may increase the risk of all-cause 
Table 3 Hazard ratios (HR) and $95 \% \mathrm{Cl}$ for all-cause mortality, according to quartile of frequency of ultra-processed food intake (times/d), among adults aged $\geq 20$ years $(n 11898$ ), Third National Health and Nutrition Examination Survey (NHANES III, 1988-1994)

\begin{tabular}{|c|c|c|c|c|c|c|c|c|c|}
\hline & \multicolumn{8}{|c|}{ Quartile of frequency of ultra-processed food intake } & \multirow[b]{3}{*}{$P$-trend } \\
\hline & \multicolumn{2}{|c|}{ Quartile 1 (n 2982) } & \multicolumn{2}{|c|}{ Quartile 2 ( $n$ 2989) } & \multicolumn{2}{|c|}{ Quartile 3 (n 2985) } & \multicolumn{2}{|c|}{ Quartile 4 (n 2942) } & \\
\hline & HR & $95 \% \mathrm{Cl}$ & $\mathrm{HR}$ & $95 \% \mathrm{Cl}$ & $\mathrm{HR}$ & $95 \% \mathrm{Cl}$ & $\mathrm{HR}$ & $95 \% \mathrm{Cl}$ & \\
\hline Deaths due to all causes, $n$ & & 625 & & 588 & & 617 & & 221 & \\
\hline Model $1^{*}$ & 1.00 & Reference & 0.98 & $0.82,1 \cdot 16$ & 1.02 & $0.83,1.24$ & 1.29 & $1.09,1.53$ & 0.002 \\
\hline Model $2 \dagger$ & 1.00 & Reference & 0.98 & $0.83,1.17$ & 1.06 & $0.85,1.29$ & 1.31 & $1.09,1.58$ & 0.001 \\
\hline Model $3 \ddagger$ & 1.00 & Reference & 0.99 & $0.83,1.18$ & 1.06 & $0.87,1.30$ & 1.30 & $1.08,1.57$ & 0.001 \\
\hline
\end{tabular}

*Model 1 was adjusted for age, sex, race/ethnicity and total energy intake.

†Model 2 was adjusted for the variables in Model 1 plus poverty level, education level, smoking status, physical activity and alcohol intake.

$\ddagger$ Model 3 was adjusted for the variables in Model 2 plus BMI, hypertension status, total cholesterol and estimated glomerular filtration rate.

Table 4 Hazard ratios (HR) and $95 \% \mathrm{Cl}$ for CVD mortality, according to quartile of frequency of ultra-processed food intake (times/d), among adults aged $\geq 20$ years ( $n 11$ 898), Third National Health and Nutrition Examination Survey (NHANES III, 1988-1994)

\begin{tabular}{|c|c|c|c|c|c|c|c|c|c|}
\hline & \multicolumn{8}{|c|}{ Quartile of frequency of ultra-processed food intake } & \multirow[b]{3}{*}{$P$-trend } \\
\hline & \multicolumn{2}{|c|}{ Quartile 1 ( $n$ 2982) } & \multicolumn{2}{|c|}{ Quartile 2 ( $n$ 2989) } & \multicolumn{2}{|c|}{ Quartile 3 ( $n$ 2985) } & \multicolumn{2}{|c|}{ Quartile 4 ( $n$ 2942) } & \\
\hline & $\mathrm{HR}$ & $95 \% \mathrm{Cl}$ & $\mathrm{HR}$ & $95 \% \mathrm{Cl}$ & $\mathrm{HR}$ & $95 \% \mathrm{Cl}$ & $\mathrm{HR}$ & $95 \% \mathrm{Cl}$ & \\
\hline Deaths due to CVD, $n$ & & 174 & & 72 & & 151 & & 51 & \\
\hline Model $1^{*}$ & 1.00 & Reference & 1.04 & $0.70,1.54$ & 0.85 & $0.56,1.28$ & 1.04 & $0.73,1.50$ & 0.88 \\
\hline Model $2 \dagger$ & 1.00 & Reference & 1.09 & $0.69,1.74$ & 0.92 & $0.60,1.41$ & $1 \cdot 10$ & $0.74,1.67$ & 0.86 \\
\hline Model $3 \ddagger$ & 1.00 & Reference & $1 \cdot 10$ & $0.69,1.76$ & 0.94 & $0.61,1.45$ & 1.13 & $0.74,1.71$ & 0.78 \\
\hline
\end{tabular}

*Model 1 was adjusted for age, sex, race/ethnicity and total energy intake.

†Model 2 was adjusted for the variables in Model 1 plus poverty level, education level, smoking status, physical activity and alcohol intake.

¥Model 3 was adjusted for the variables in Model 2 plus BMI, hypertension status, total cholesterol and estimated glomerular filtration rate.

mortality. Participants in the highest quartile of frequency of ultra-processed food intake consumed higher amounts of total energy, total fat, saturated fat and added sugar, and lower amounts of protein, micronutrients and fibre. Low dietary fibre and micronutrients may contribute to earlier death by increasing the risk of major chronic conditions and damaging mitochondrial function ${ }^{(40,41)}$. In addition, those in the highest quartile had poor overall diet quality and nutritional quality. In epidemiological studies, diet quality has been shown to be a strong predictor of incident chronic conditions such as diabetes, CVD, cancer and allcause mortality ${ }^{(29,42-44)}$. However, when we further adjusted for diet quality scores, the results on ultraprocessed food intake and all-cause mortality did not change. This suggests that diet quality may not be a strong mediator of the association between ultra-processed food intake and mortality and there may be other bioactive compounds in ultra-processed foods that can increase the risk of death ${ }^{(23)}$.

Ultra-processed food intake may elevate the risk of allcause mortality by increasing exposure to contaminants and environmental chemicals. For example, acrylamide is formed during heating ${ }^{(13)}$, and this compound is considered by the US Environmental Protection Agency and the National Toxicology Program as a neurotoxin and carcinogenic $^{(45)}$. Furthermore, environmental chemicals that are present in food packaging, such as phthalates and bisphenol A, have been shown to be associated with adverse health outcomes ${ }^{(11,46,47)}$. Phthalates and bisphenol $\mathrm{A}$ are endocrine disruptors that are associated with diabetes and obesity in cross-sectional studies ${ }^{(15,16,48)}$. Examining environmental chemicals in ultra-processed foods was beyond the scope of our study, and chemical exposure biomarkers were not available at NHANES III. Further investigation on environmental chemicals in ultraprocessed foods and health outcomes is necessary to test these hypotheses.

Given the association between ultra-processed food consumption and CVD risk factors ${ }^{(21,22)}$, the null association with CVD mortality in our study was surprising. However, it is known that cause-of-death information is often inaccurate on death certificates for $\mathrm{CVD}^{(49,50)}$. In our data set, the most common cause of death was other causes. It is possible that deaths were coded as 'other' if the underlying cause of death was not apparent. In addition, individuals with CVD may be less likely to die from this condition since there are many effective treatments for reducing CVD risk factors such as blood pressure and cholesterol. We had a smaller number of cause-specific (cardiovascular) deaths and therefore less power to detect significant associations. Additional research investigating the association of ultra-processed food intake with CVD events and mortality is needed.

Another surprising result was a lower intake of $\mathrm{Na}$ at higher reported frequency of ultra-processed food intake. Given the common notion that processed foods are the 
largest sources of $\mathrm{Na}$ intake ${ }^{(51)}$, we expected to find higher $\mathrm{Na}$ intake in this group. However, similar to our results, a cross-sectional study that used two dietary recalls from NHANES 2009-2010 found lower Na intake among those in the highest quintile of ultra-processed food consumption $^{(8)}$. In contrast, a study in France - which used a specific module to include $\mathrm{Na}$ naturally present in foods, salt added during food preparation and at the table, and validated it against urinary $\mathrm{Na}$ excretion - showed that $\mathrm{Na}$ intake was the highest among those in the highest intake quartile of ultra-processed foods ${ }^{(23)}$. Lower $\mathrm{Na}$ intake observed in our study may be due to not accounting for salt added during preparation or at the table in NHANES III or possibly underestimation of this nutrient in the food composition database.

Strengths of our study include the use of nationally representative data of US adults, long-term follow-up period, minimal loss to follow-up ( $0 \cdot 1 \%)$, predefined criteria to classify food items by degree of processing, and comprehensive nutrient analyses considering overall nutritional quality and diet quality.

Several limitations need to be considered. The FFQ that was used in the present study was not designed to answer the research question on food processing. Thus, several ultra-processed foods such as mass-produced breads were not included. Further, there is potential for misclassification. For example, we classified breads as processed foods, but consumption of mass-produced breads, which is considered an ultra-processed food, may be more common than consumption of artisanal breads or handmade breads. We did not use participants' $24 \mathrm{~h}$ dietary recall data to rank participants by ultra-processed food consumption because the $24 \mathrm{~h}$ dietary recall does not represent usual intakes of foods and beverages ${ }^{(52)}$. Only $5 \%$ of the participants completed a second $24 \mathrm{~h}$ dietary recall in this data set, making it difficult to reliably distinguish between those who consumed ultra-processed foods frequently, infrequently and never ${ }^{(53,54)}$. Moreover, in NHANES III, the $24 \mathrm{~h}$ dietary recall does not provide detailed descriptions of foods and beverages. This is problematic, particularly for recipe foods, because the data set does not have detailed ingredient lists and focuses mainly on fats used for cooking and fat content of protein foods. Unfortunately, the $24 \mathrm{~h}$ dietary recall does not provide better information than the FFQ in NHANES III to classify foods according to different levels of processing. The classification of foods by processing level using the $24 \mathrm{~h}$ dietary recall would require making assumptions about ingredients, which would be subjective and may lead to misclassification.

Another limitation is that no validation study was conducted for the specific FFQ used in NHANES III. However, similar questionnaires of varying length have been validated $^{(55,56)}$. In addition, there was a similar trend of demographic characteristics and macro- and micronutrient intakes according to quartiles of ultra-processed food intake when we compared them with studies that used a semi-quantitative FFQ and series of $24 \mathrm{~h}$ dietary recalls ${ }^{(21-23)}$. This suggests that the FFQ used in our study ranked participants into different quartiles reasonably well. Because frequency of ultra-processed food consumption is not necessarily the only or best proxy for dietary contribution of ultra-processed foods, it is important for future studies to confirm our findings using quantitative estimates of absolute intake instead of frequency of consumption of ultra-processed foods. Next, information bias may be a limitation. Participants may have under-reported ultra-processed food intake at baseline because of social stigma and this could have led to an underestimation of the associations. However, information bias may not be a concern in our data because participants were not specifically asked about ultra-processed food consumption. Participants reported their dietary intakes using an FFQ, and we used prespecified criteria to classify foods according to different levels of processing. Another limitation is that the NHANES III was conducted several decades ago and thus food intake assessed in this cycle does not reflect the current US food supply. Participants' dietary intake was measured only at baseline, because repeated assessment was not available in this data set. Considering that the modern food supply has shifted to include more ultra-processed foods than in 1988-1994 ${ }^{(1)}$, it is important to replicate our findings in more recent settings where there are repeated measurements of diet to better understand the association between ultra-processed foods and long-term health outcomes. Lastly, even though we controlled for the most important confounders including sociodemographic characteristics, health behaviours and potential risk factors for mortality, there is a possibility of residual confounding due to unmeasured or incorrectly measured covariates.

In addition to these considerations, it may be important to consider the issue of self-selection in future studies, because it is possible that those who were more at risk for death had poorer self-care in general, beyond their dietary choices. However, when we examined additional variables in the NHANES III, we did not find evidence that those in the highest quartile of ultra-processed food intake had poorer self-care. The NHANES III did not assess factors that may have influenced participants to consume ultraprocessed foods (i.e. knowledge of potential adverse health outcomes associated with ultra-processed food consumption, perceived convenience, taste and price of ultra-processed foods, or the food environment), which can provide important information to address self-selection. Future studies on ultra-processed foods and health outcomes should consider collecting this information.

\section{Conclusion}

In conclusion, we found an elevated risk of all-cause mortality in association with higher frequency of 
ultra-processed food intake in a nationally representative sample of US adults. Given the consistent results on elevated health risk of ultra-processed food intake, future dietary guidelines might consider making a recommendation about degree of food processing. More longitudinal studies with dietary data reflecting the modern food supply are needed to confirm our results.

\section{Acknowledgements}

Financial support: C.M.R. was supported by a Mentored Research Scientist Development Award from the National Institute of Diabetes and Digestive and Kidney Diseases (grant number K01 DK107782). The National Institute of Diabetes and Digestive and Kidney Diseases had no role in the design, analysis or writing of this article. Conflict of interest: All authors have indicated they have no conflicts of interest or financial disclosures relevant to this article. Authorship: H.K. drafted the manuscript and analysed the data. E.A.H. assisted with statistical analyses, interpreted the data and provided critical feedback. C.M.R. designed the research and was involved in all aspects of the manuscript from analyses to writing. All authors read and approved the final manuscript. Ethics of human subject participation: All data were obtained from secondary sources and available publicly. No protocol approval was necessary.

\section{Supplementary material}

To view supplementary material for this article, please visit https://doi.org/10.1017/S1368980018003890

\section{References}

1. Monteiro CA, Moubarac J-C, Cannon G et al. (2013) Ultraprocessed products are becoming dominant in the global food system. Obes Rev 14, 21-28.

2. Steele EM, Baraldi LG, Louzada ML da C et al. (2016) Ultraprocessed foods and added sugars in the US diet: evidence from a nationally representative cross-sectional study. BMJ Open 6, e009892.

3. Moubarac J-C, Batal M, Louzada ML et al. (2017) Consumption of ultra-processed foods predicts diet quality in Canada. Appetite 108, 512-520.

4. Louzada ML da C, Ricardo CZ, Steele EM et al. (2018) The share of ultra-processed foods determines the overall nutritional quality of diets in Brazil. Public Health Nutr 21, 94-102.

5. Julia C, Martinez L, Allès B et al. (2018) Contribution of ultra-processed foods in the diet of adults from the French NutriNet-Santé study. Public Health Nutr 21, $27-37$.

6. Marrón-Ponce JA, Sánchez-Pimienta TG, Louzada ML da C et al. (2018) Energy contribution of NOVA food groups and sociodemographic determinants of ultra-processed food consumption in the Mexican population. Public Health Nutr 21, 87-93.
7. Cediel G, Reyes M, Louzada ML da C et al. (2018) Ultraprocessed foods and added sugars in the Chilean diet (2010). Public Health Nutr 21, 125-133.

8. Martínez Steele E, Popkin BM, Swinburn B et al. (2017) The share of ultra-processed foods and the overall nutritional quality of diets in the US: evidence from a nationally representative cross-sectional study. Popul Health Metr $15,6$.

9. Costa CS, Del-Ponte B, Assunção MCF et al. (2018) Consumption of ultra-processed foods and body fat during childhood and adolescence: a systematic review. Public Health Nutr 21, 148-159.

10. Heindel JJ, Blumberg B, Cave M et al. (2017) Metabolism disrupting chemicals and metabolic disorders. Reprod Toxicol 68, 3-33.

11. Zota AR, Phillips CA \& Mitro SD (2016) Recent fast food consumption and bisphenol $\mathrm{A}$ and phthalates exposures among the US population in NHANES, 2003-2010. Environ Health Perspect 124, 1521-1528.

12. US Environmental Protection Agency (2013) Learn about the Toxics Release Inventory. https://www.epa.gov/toxicsrelease-inventory-tri-program/learn-about-toxics-releaseinventory (accessed April 2018).

13. Nerín C, Aznar M \& Carrizo D (2016) Food contamination during food process. Trend Food Sci Technol 48, 63-68.

14. Birlouez-Aragon I, Morales F, Fogliano V et al. (2010) The health and technological implications of a better control of neoformed contaminants by the food industry. Pathol Biol 58, 232-238.

15. Stahlhut RW, van Wijngaarden E, Dye TD et al. (2007) Concentrations of urinary phthalate metabolites are associated with increased waist circumference and insulin resistance in adult US males. Environ Health Perspect 115, 876-882.

16. Liu B, Lehmler H-J, Sun Y et al. (2017) Bisphenol A substitutes and obesity in US adults: analysis of a populationbased, cross-sectional study. Lancet Planet Health 1, e114-e122.

17. Rauber F, Campagnolo PDB, Hoffman DJ et al. (2015) Consumption of ultra-processed food products and its effects on children's lipid profiles: a longitudinal study. Nutr Metab Cardiovasc Dis 25, 116-122.

18. Louzada ML da C, Baraldi LG, Steele EM et al. (2015) Consumption of ultra-processed foods and obesity in Brazilian adolescents and adults. Prev Med 81, 9-15.

19. Canella DS, Levy RB, Martins APB et al. (2014) Ultraprocessed food products and obesity in Brazilian households (2008-2009). PLoS One 9, e92752.

20. Juul F, Martinez-Steele E, Parekh N et al. (2018) Ultraprocessed food consumption and excess weight among US adults. Br J Nutr 120, 90-100.

21. Mendonça R de D, Pimenta AM, Gea A et al. (2016) Ultraprocessed food consumption and risk of overweight and obesity: the University of Navarra Follow-Up (SUN) cohort study. Am J Clin Nutr 104, 1433-1440.

22. Mendonça R de D, Lopes ACS, Pimenta AM et al. (2017) Ultra-processed food consumption and the incidence of hypertension in a Mediterranean cohort: the Seguimiento Universidad de Navarra Project. Am J Hypertens 30, 358-366.

23. Fiolet T, Srour B, Sellem L et al. (2018) Consumption of ultra-processed foods and cancer risk: results from NutriNetSanté prospective cohort. BMJ 360, k322.

24. National Center for Health Statistics (1994) Plan and operation of the Third National Health and Nutrition Examination Survey, 1988-94. Series 1: programs and collection procedures. Vital Health Stat 1, issue 32, 1-407.

25. Monteiro CA, Cannon G, Moubarac J-C et al. (2018) The UN Decade of Nutrition, the NOVA food classification and the trouble with ultra-processing. Public Health Nutr 21, 5-17. 
26. Pan American Health Organization (2015) Ultra-Processed Food and Drink Products in Latin America: Trends, Impact on Obesity, Policy Implications. Washington, DC: PAHO.

27. National Cancer Institute (2018) Pyramid Servings Database (PSDB) for NHANES III: overview. https://epi.grants.cancer. gov/pyramid/ (accessed April 2018).

28. Fulgoni VL, Keast DR \& Drewnowski A (2009) Development and validation of the Nutrient-Rich Foods index: a tool to measure nutritional quality of foods. J Nutr 139, 1549-1554.

29. Streppel MT, Sluik D, van Yperen JF et al. (2014) Nutrientrich foods, cardiovascular diseases and all-cause mortality: the Rotterdam study. Eur J Clin Nutr 68, 741-747.

30. Drewnowski A (2009) Defining nutrient density: development and validation of the nutrient rich foods index. $J \mathrm{Am}$ Coll Nutr 28, issue 4, 421S-426S.

31. National Center for Health Statistics (2000) Third National Health and Nutrition Examination Survey, 1988-1994: NHANES III Healthy Eating Index Data File (Series 11, No. 6A). Hyattsville, MD: Centers for Disease Control and Prevention.

32. National Center for Health Statistics (2013) NCHS 2011 linked mortality files matching methodology. https://www. cdc.gov/nchs/data/datalinkage/2011_linked_mortality_file_ matching_methodology.pdf (accessed April 2018).

33. World Health Organization (1992) International Classification of Diseases and Related Health Problems, 10th Revision. Geneva: WHO.

34. Ainsworth BE, Haskell WL, Whitt MC et al. (2000) Compendium of physical activities: an update of activity codes and MET intensities. Med Sci Sports Exerc 32, 9 Suppl., S498-S504.

35. Allain CC, Poon LS, Chan CS et al. (1974) Enzymatic determination of total serum cholesterol. Clin Chem 20 , 470-475.

36. Levey AS, Stevens LA, Schmid $\mathrm{CH}$ et al. (2009) A new equation to estimate glomerular filtration rate. Ann Intern Med 150, 604-612.

37. Selvin E, Manzi J, Stevens LA et al. (2007) Calibration of serum creatinine in the National Health and Nutrition Examination Surveys (NHANES) 1988-1994, 1999-2004. Am J Kidney Dis 50, 918-926.

38. Rao JNK \& Scott AJ (1987) On simple adjustments to chisquare tests with sample survey data. Ann Stat 15, 385-397.

39. Schwingshackl L, Schwedhelm C, Hoffmann G et al. (2017) Food groups and risk of all-cause mortality: a systematic review and meta-analysis of prospective studies. Am J Clin Nutr 105, 1462-1473

40. Yang Y, Zhao L-G, Wu Q-J et al. (2015) Association between dietary fiber and lower risk of all-cause mortality: a meta-analysis of cohort studies. Am J Epidemiol 181, 83-91.

41. Ames BN (2006) Low micronutrient intake may accelerate the degenerative diseases of aging through allocation of scarce micronutrients by triage. Proc Natl Acad Sci US A 103, 17589-17594.

42. Chiuve SE, Sampson L \& Willett WC (2011) Adherence to the overall nutritional quality index and risk of total chronic disease. Am J Prev Med 40, 505-513.

43. Rathod AD, Bharadwaj AS, Badheka AO et al. (2012) Healthy Eating Index and mortality in a nationally representative elderly cohort. Arch Intern Med 172, 275-277.

44. Lassale C, Gunter MJ, Romaguera D et al. (2016) Diet quality scores and prediction of all-cause, cardiovascular and cancer mortality in a pan-European cohort study. PLoS One 11, e0159025.

45. Centers for Disease Control and Prevention (2018) Public health statement for acrylamide. https://www.atsdr. cdc.gov/phs/phs.asp?id=1113\&tid=236 (accessed April 2018).

46. Rudel RA, Gray JM, Engel CL et al. (2011) Food packaging and bisphenol A and bis(2-ethyhexyl) phthalate exposure: findings from a dietary intervention. Environ Health Perspect 119, 914-920.

47. Varshavsky JR, Morello-Frosch R, Woodruff TJ et al. (2018) Dietary sources of cumulative phthalates exposure among the US general population in NHANES 2005-2014. Environ Int 115, 417-429.

48. James-Todd T, Stahlhut R, Meeker JD et al. (2012) Urinary phthalate metabolite concentrations and diabetes among women in the National Health and Nutrition Examination Survey (NHANES) 2001-2008. Environ Health Perspect 120, 1307-1313.

49. Ravakhah K (2006) Death certificates are not reliable: revivification of the autopsy. South Med J 99, 728-733.

50. Smith CJ, Scott SM \& Wagner BM (1998) The necessary role of the autopsy in cardiovascular epidemiology. Hum Pathol 29, 1469-1479.

51. Brown IJ, Tzoulaki I, Candeias V et al. (2009) Salt intakes around the world: implications for public health. Int J Epidemiol 38, 791-813.

52. Willett WC (2012) Nutritional Epidemiology, 3rd ed. Oxford/New York: Oxford University Press.

53. National Cancer Institute (2018) Usual dietary intakes: the NCI method. https://epi.grants.cancer.gov/diet/usualintakes/ method.html (accessed November 2018).

54. National Center for Health Statistics (1999) NHANES III Second Exam File Documentation (Series 11, No. 3A). Hyattsville, MD: Centers for Disease Control and Prevention.

55. Willett WC, Sampson L, Stampfer MJ et al. (1985) Reproducibility and validity of a semiquantitative food frequency questionnaire. Am J Epidemiol 122, 51-65.

56. Subar AF, Dodd KW, Guenther PM et al. (2006) The food propensity questionnaire: concept, development, and validation for use as a covariate in a model to estimate usual food intake. J Am Diet Assoc 106, 1556-1563. 